\title{
Aleitamento materno e perfil sociodemográfico e obstétrico entre puérperas atendidas em maternidade pública de referência
}

\author{
Breastfeeding and sociodemographic and obstetric profile among puérperas attended in public \\ maternity of reference \\ Lactancia materna y perfil sociodemográfico y obstétrico entre las puérperas atendidas en \\ maternidad pública de referencia
}

\section{Resumo}

Objetivo: conhecer o histórico do aleitamento materno, o perfil sociodemográfico e obstétrico entre puérperas atendidas em maternidade pública de referência. Metodologia: Estudo em campo, transversal com abordagem quantitativa, desenvolvido entre novembro de 2019 e março de 2020, com 300 puérperas internadas em uma maternidade pública de referência, selecionadas por conveniência. Dados coletados em formulário contendo perguntas acerca de informações sociodemográficas, obstétricas e de amamentação e analisados de forma descritiva considerando as frequências absolutas e relativas das variáveis. Pesquisa aprovada pelo Comitê de Ética em Pesquisa da Universidade Federal do Maranhão sob o protocolo no 3.450.563. Resultados: As puérperas tinham idade entre 18 e 30 anos; $61,0 \%$ eram casadas ou em união estável; 72,6\% cursaram o Ensino Médio; 65,4\% com renda familiar entre 1 e 2 salários mínimos; 76,0\% eram donas de casa; 42,7\% tinham 2 filhos; 99,7\% fizeram pré-natal; 65,4\% fizeram seis ou mais consultas; 49,3\% foram orientadas sobre a prática da amamentação durante o pré-natal; 76,7\% já amamentaram anteriormente; 60,0\% não tiveram dificuldades para amamentar e as demais referiram queixa de pouco leite $(22,0 \%)$, fissura no mamilo $(15,4 \%)$, se queixaram de ter leite fraco $(1,6 \%)$ e sentiram dor $(1,0 \%)$. Conclusão: Evidenciou-se a importância do uso de atividades educativas voltadas para a amamentação pelos profissionais de saúde que prestam assistência ao pré-natal, parto e puerpério, visando contribuir para que as mães tenham condições de amamentar, reduzindo assim as taxas de desmame precoce.

Palavras-chave: Aleitamento materno; Puerpério; Desmame precoce; Lactante.

\footnotetext{
Abstract

Objective: was to know the history of breastfeeding, the sociodemographic and obstetric profile among postpartum women attended in a reference public maternity hospital. Methodology: cross-sectional field study with quantitative
} 
approach, developed between November 2019 and March 2020, with 300 puerpery hospitalized in a reference public maternity hospital, selected for convenience. Data collected in a form containing questions about sociodemographic, obstetric and breastfeeding information and analyzed descriptively considering the absolute and relative frequencies of the variables. Research approved by the Research Ethics Committee of the Federal University of Maranhão under protocol no. 3,450,563. Results: The puerpery women were between 18 and 30 years old; $61.0 \%$ were married or in a stable union; $72.6 \%$ attended high school; $65.4 \%$ with family income between 1 and 2 minimum wages; $76.0 \%$ were housewives; $42.7 \%$ had 2 children; $99.7 \%$ had prenatal care; $65.4 \%$ had six or more consultations; $49.3 \%$ were oriented about breastfeeding during prenatal care; $76.7 \%$ have breastfed before; $60.0 \%$ had no difficulties to breastfeed and the others reported a complaint of little milk $(22.0 \%)$, nipple fissure $(15.4 \%)$, complained of having weak milk (1.6\%) and felt pain (1.0\%). Conclusion: It was evidenced the importance of the use of educational activities aimed at breastfeeding by health professionals who provide prenatal, childbirth and puerperium care, aiming to contribute to the mothers being able to breastfeed, thus reducing the rates of early weaning.

Keywords: Breastfeeding; Puerperium; Early weaning; Lactating.

\begin{abstract}
Resumen
Objetivo: conocer la história de la lactancia materna, el perfil sociodemográfico y obstétrico entre las mujeres posparto atendidas en un hospital público de maternidad de referência. Metodologia: Estudio de campo transversal con enfoque cuantitativo, desarrollado entre noviembre de 2019 y marzo de 2020, con 300 puerperios hospitalizados en una maternidad pública de referencia, seleccionados por conveniencia. Datos recolectados en un formulario que contiene preguntas sobre información sociodemográfica, obstétrica y de lactancia materna y analizados descriptivamente considerando las frecuencias absolutas y relativas de las variables. Investigación aprobada por el Comité de Ética en Investigación de la Universidad Federal de Maranhão bajo el protocolo no 3.450.563. Resultados: Las mujeres puerperiódricas tenían entre 18 y 30 años; el 61,0\% estaban casados o en una unión estable; el 72,6\% asistió a la escuela secundaria; 65,4\% con ingresos familiares entre 1 y 2 salarios mínimos; el 76,0\% eran amas de casa; el 42,7\% tuvo 2 hijos; el 99,7\% tenía atención prenatal; el 65,4\% tuvo seis o más consultas; el 49,3\% se orientó sobre la lactancia materna durante la atención prenatal; el 76,7\% ha amamantado antes; El 60,0\% no tuvo dificultades para amamantar y los demás reportaron una queja de poca leche $(22,0 \%)$, fisura del pezón $(15,4 \%)$, se quejaron de tener leche débil $(1,6 \%)$ y sintieron dolor $(1,0 \%)$. Conclusión: Se evidenció la importancia del uso de actividades educativas dirigidas a la lactancia materna por parte de los profesionales de la salud que brindan atención prenatal, de parto y puerperio, con el objetivo de contribuir a que las madres puedan amamantar, reduciendo así las tasas de destete precoz.
\end{abstract}

Palabras clave: Lactancia materna; Puerperio; Destete temprano; Lactantes.

\title{
1. Introdução
}

O ciclo gravídico puerperal é um período crítico na vida da mulher, onde há transformações fisiológicas, físicas e psicológicas, e em seu papel social, o que exige adaptações interpessoais, visto que essas transformações podem afetar o seu cotidiano bem como das pessoas que a cercam e com ela convivem. As modificações fisiológicas e psicológicas que acometem a mulher podem gerar desequilíbrio que interferem na sua vida (Alves et al., 2020).

Conhecer as características sociodemográficas das mulheres pode contribuir para uma abordagem de saúde integral e significativa, considerando que cada uma tem um contexto social, enfrenta desafios pessoais, e vive sua própria realidade, de forma que a assistência dessa gestante no pré-natal e puerpério estejam voltadas para minimizar os agravos, de forma que conhecer essas características pode direcionar as atividades de cuidado e de assistência visando a prevenção de riscos que envolvem a gestante/puérpera e o tratamento de agravos, estabelecendo um cuidado individualizado e humanizado (Amorim et al., 2017).

A faixa etária, a raça/cor, escolaridade, renda familiar, presença ou ausência de um companheiro, pode influenciar no acesso a assistência à saúde, considerando que ações de promoção da saúde por meio de intervenções clínicas e educativas contribuem para o conhecimento de melhores práticas que coadunam para uma melhor qualidade de vida (Moraes et al., 2020).

Diante dessas considerações, acredita-se que as ações do profissional de saúde são importantes no pré-natal, uma vez que por meio da assistência prestada, é possível identificar intercorrências precocemente e monitorar as gestantes que se encontram em situação de risco (Reis et al., 2017).

Um acolhimento adequado dos profissionais de saúde pode trazer mais segurança à gestante diante das descobertas advindas em cada semana de gestação, proporcionando assim, uma gravidez mais segura. Um atendimento adequado ao pré- 
natal é composto por ações individualizadas visando atender as necessidades da mulher, com responsabilização e formação de vínculo, a escuta ativa dos problemas por ela enfrentados, com troca de informações, possibilitando intervenções eficazes e pertinentes para atender as suas necessidades no momento adequado (Barbosa et al., 2020).

Não só durante o pré-natal a assistência a saúde é importante, mas sobretudo durante o parto e puerpério. É nessa fase que a mulher pode ter algumas dificuldades para desempenhar o seu papel de mãe, o que requer algumas adaptações para a instrumentalização dos cuidados com o recém-nascido. Entre os cuidados mais importantes está o aleitamento materno (AM) (Rodrigues et al., 2016)

O AM é considerado necessário para a manutenção da saúde do bebê, sendo completo em nutrientes e a melhor fonte nutricional, além de atuar no sistema imunológico sendo considerado como a primeira vacina que protege a saúde do bebê (Melo et al., 2021).

Apesar dos benefícios comprovados da amamentação, a taxa de adesão a essa prática está abaixo do preconizado, em especial o aleitamento materno exclusivo (AME). O aleitamento deve ser exclusivo nos primeiros seis meses, seguido pela introdução de alimentos complementares após esse momento, e a amamentação deve ser mantida até os dois anos de vida da criança ou mais, segundo recomendações da Organização Mundial da Saúde (OMS), Fundo das Nações Unidas para a Infância (UNICEF), e Academia Americana de Pediatria (OMS, 2019).

No Brasil, apenas $41 \%$ das crianças menores de seis meses foram amamentadas exclusivamente por leite materno (Brasil, 2020). As primeiras horas após o parto são consideradas críticas para o estabelecimento de uma amamentação efetiva. O manejo adequado da lactação contribui para a melhoria dos indicadores de AME. Entretanto, é primordial promover a autoconfiança da mãe contribuindo para diminuir as dificuldades que possam acontecer (Harari et al., 2018). As principais dificuldades citadas pelas mães ao amamentar são: leite insuficiente, introdução precoce de leite artificial (Freitas, et al., 2018), o trabalho materno fora do lar, leite fraco, pega incorreta, desconhecimento dos benefícios da amamentação (Sousa et al., 2017).

Tendo em vista uma melhor assistência a saúde da mulher, considerando que o local de realização da pesquisa é uma maternidade de referência regional, o presente estudo foi desenvolvido com o objetivo de conhecer o histórico do aleitamento materno, o perfil sociodemográfico e obstétrico entre puérperas atendidas em maternidade pública de referência.

\section{Metodologia}

Estudo em campo, transversal com abordagem quantitativa desenvolvido no período de novembro de 2019 a março de 2020, com puérperas internadas em uma maternidade pública de referência regional situada no sudoeste maranhense. O método quantitativo permite a análise por técnicas matemáticas (Pereira et al., 2018). "Já os estudos em campo contam com muitas variáveis e a coleta é feita em condições reais" (Pereira et al., 2018, p. 101).

O estudo foi realizado com mulheres que estavam no puerpério imediato, internadas nas enfermarias da maternidade. Considerando que mensalmente são realizados em média 400 procedimentos entre partos normais e cesarianas, com um intervalo com $95 \%$ de confiança, a amostra compreendeu 300 participantes.

As participantes foram selecionadas por amostragem não probabilística por conveniência, a partir dos seguintes critérios de inclusão: estar no puerpério imediato acompanhadas de seus bebês, com qualquer idade, que tinham condições de se comunicar com os pesquisadores, independente do tipo de parto. Foram excluídas as puérperas sem instrução ou analfabetas, as que possuíam alguma doença que contraindicasse a amamentação, as que tinham seus bebês internados na Unidade de Terapia Intensiva e as com deficiência visual ou auditiva.

Os dados foram coletados por um único pesquisador, sendo utilizado um formulário elaborado para a presente pesquisa contendo perguntas acerca de informações sociodemográficas (idade, estado civil, escolaridade, renda familiar e 
situação laboral), obstétricas (número de filhos, se fez pré-natal e número de consultas) e de amamentação (se recebeu orientação sobre AM no pré-natal, se já amamentou anteriormente, se praticou o aleitamento materno exclusivo (AME) e dificuldades na prática da amamentação).

A puérpera recebeu um convite verbal, individual, para participar da pesquisa no momento que estava internada na enfermaria, era explicado como aconteceria sua participação e as eventuais dúvidas foram esclarecidas. Em seguida a mesma assinava o Termo de Consentimento Livre e Esclarecido (TCLE) concordando com sua participação.

Os dados foram analisados de forma descritiva considerando as frequências absolutas e relativas das variáveis utilizando as planilhas do programa Microsoft Excel.

A presente pesquisa atendeu aos preceitos éticos de acordo com a Resolução No 466 de 2012 (Brasil, 2012) que trata de pesquisas e testes em seres humanos e foi aprovada pelo Comitê de Ética em Pesquisa da Universidade Federal do Maranhão sob o protocolo no 3.450.563.

\section{Resultados}

No período sob investigação, 300 puérperas participaram da pesquisa e na Tabela 1 são apresentados os dados sobre as características sociodemográficas das mesmas.

Tabela 1 - Distribuição dos dados sociodemográficos de puérperas internadas em enfermarias, Hospital Regional Materno Infantil. Imperatriz, MA, 2020.

\begin{tabular}{lll}
\hline Características sociodemográficas & $\mathbf{n}$ & $\%$ \\
\hline Idade & 21 & 7,0 \\
$<18$ & 261 & 87,0 \\
18 a 30 anos & 18 & 6,0 \\
31 a 40 anos & & \\
Estado Civil & 183 & 61,0 \\
Casada/União estável & 116 & 38,7 \\
Solteira & 1 & 0,3 \\
Divorciada/separada & & \\
Escolaridade & 60 & 20,0 \\
Ensino Fundamental & 218 & 72,6 \\
Ensino Médio & 22 & 7,4 \\
Ensino Superior & & \\
Renda familiar (salário mínimo*) & 92 & 30,7 \\
$\leq 1$ salário mínimo & 201 & 67,0 \\
1 a 2 salários mínimos & 7 & 2,3 \\
$\geq 3$ salários mínimos & & \\
Situação laboral & 72 & 24,0 \\
Trabalha fora de casa & 228 & 76,0 \\
Dona de casa & 300 & 100 \\
\hline Total & & \\
\hline
\end{tabular}

Fonte: Autores (2020). *Salário mínimo vigente R \$ 1.045,00. 
No que diz respeito à caracterização do perfil sociodemográfico das 300 puérperas avaliadas, observou-se que a maioria tinha idade entre 18 e 30 anos (261: 87,0\%); era casada ou em união estável (183: 61,0\%); cursou o Ensino Médio (218: 72,6\%); com renda familiar entre 1 e 2 salários mínimos (201: 67,0\%) e eram donas de casa (228: 76,0\%) (Tabela 1).

O perfil de obstétrico das participantes do estudo pode ser observado na Tabela 2.

Tabela 2 - Distribuição dos dados obstétricos de puérperas internadas em enfermarias, Hospital Regional Materno Infantil. Imperatriz, MA, 2020.

\begin{tabular}{lll}
\hline Características obstétricas & $\mathbf{n}$ & $\mathbf{\%}$ \\
\hline Número de filhos & 70 & 23,3 \\
1 filho & 128 & 42,7 \\
2 filhos & 100 & 33,3 \\
3 a 5 filhos & 2 & 0,7 \\
$>$ < filhos & & \\
Fez pré-natal & 299 & 99,7 \\
Sim & 1 & 0,3 \\
Não & & \\
Número consultas & 103 & 34,3 \\
$<6$ & 196 & 65,4 \\
6 ou mais & 1 & 0,3 \\
Nenhuma & 300 & 100 \\
\hline Total & & \\
\hline
\end{tabular}

Fonte: Autores (2020).

Sobre o perfil obstétrico das participantes, grande parte delas tinha 2 filhos (128: 42,7\%), fizeram pré-natal (299: $99,7 \%$ ), fizeram seis ou mais consultas (196: 65,4\%) (Tabela 2).

Em relação aos dados de amamentação das participantes, estão descritos na Tabela 3. 
Tabela 3 - Distribuição dos dados sobre o histórico do aleitamento materno de puérperas internadas em enfermarias, Hospital Regional Materno Infantil. Imperatriz, MA, 2020.

\begin{tabular}{lll}
\hline Características de amamentação & n & \% \\
\hline Orientação sobre amamentação no pré-natal & 148 & 49,3 \\
Sim & 152 & 50,7 \\
Não & & \\
Já amamentou antes & 230 & 76,7 \\
Sim & 70 & 23,3 \\
Não & & \\
Tempo de AME* & 16 & 5,4 \\
$\leq 1$ mês & 72 & 24,0 \\
2 a 3 meses & 67 & 22,3 \\
4 a 5 meses & 75 & 25,0 \\
6 meses & 70 & 23,3 \\
Não amamentou & & \\
Dificuldades para amamentar & 46 & 15,4 \\
Fissura no mamilo & 66 & 22,0 \\
Pouco leite & 5 & 1,6 \\
Leite fraco & 3 & 1,0 \\
Dor & 180 & 60,0 \\
Sem dificuldades & 300 & 100 \\
\hline Total & 30 \\
\hline
\end{tabular}

Fonte: Autores (2020). *AME Aleitamento materno exclusivo.

Em relação aos dados sobre as características da amamentação, (148: 49,3\%) foram orientadas sobre a prática durante o pré-natal ao passo que (152: 50,7\%) não receberam orientação sobre amamentação no pré-natal; já amamentaram anteriormente (230: 76,7\%); praticaram AME até seis meses (75: 25,0\%), entre 4 e 5 meses (67: 22,3\%), de 2 a 3 meses (72: 24,0\%) e (16: 5,4\%) menos de 1 mês; não tiveram dificuldades para amamentar (180: 60,0\%) e as demais referiram queixa de pouco leite (66: $22,0 \%)$, fissura no mamilo (46: $15,4 \%)$, se queixaram de ter leite fraco $(5: 1,6 \%)$ e sentiram dor $(3: 1,0 \%)$ (Tabela 3).

\section{Discussão}

O AM tem sido amplamente discutido atualmente sendo imperativo investigar os fatores que envolvem essa prática, inclusive os relacionados ao perfil sociodemográfico das lactantes (Alves et al., 2021).

A idade materna maior pode representar um fator de proteção ao AM (Vila-Candel et al., 2019). Os achados neste estudo revelaram o predomínio de mulheres jovens nessa populacão, sendo a faixa etária mais frequente entre 18 e 30 anos, entre $87 \%$ das mães. Estudo realizado em Belém (PA) demonstrou que as mulheres com idade superior a 30 anos possuem mais conhecimento o que contribui para uma prática mais duradoura de AM, o que significa uma proteção a amamentação (Margotti et al., 2017).

No presente estudo $61 \%$ das participantes eram casadas ou vivia em união estável, o que é considerado um aspecto positivo, visto que, o apoio e incentivo do companheiro são fundamentais para a mulher que amamenta. Estudo realizado no 
Mato Grosso do Sul demonstrou que a presença do parceiro favorece a amamentação, entretanto, identificaram que a quantidade de pais que não receberam orientações sobre amamentação é preocupante, demonstrando que a participação dos profissionais responsáveis pelo pré-natal é fundamental nesse processo de orientação e inclusão paterna (Lima et al., 2017).

A participação dos companheiros durante a amamentação é prazerosa e contribui para que se estabeleça o apoio a lactante. Na rede social essa é uma influência positiva para a amamentação, em especial ao AME, de modo que sua ausência pode representar um fator contribuinte para o desmame precoce (Alves et al., 2018).

Quanto a escolaridade, a maioria das pesquisadas (72,6\%) cursaram o ensino médio. Mulheres que tem mais de oito anos de estudo provavelmente conhecem melhor os benefícios do AME, o alto valor nutritivo e calórico do leite materno, nutrientes essenciais para o crescimento e desenvolvimento do bebê. Uma escolaridade materna menor influencia a introdução prematura de leite artificial o que leva a redução do AME, no entanto, um nível mais elevado de instrução da mãe representa um fator positivo a amamentação (Margotti et al., 2017).

Estudo realizado em Recife (PE) mostrou que a maior parte da população estudada tinha baixa escolaridade e não possuía nenhum tipo de renda o que são fatores que podem influenciar na interrupção do aleitamento materno, considerando que mulheres que tem mais acesso a informação podem compreender melhor a importância e os benefícios da amamentação (Rocha et al., 2018).

A renda familiar de $67 \%$ das participantes variou de um a dois salários mínimos, o que pode ser considerado um fator limitador, visto que o nível de conhecimento sobre amamentação pode estar associado à renda familiar. Mães com renda maior tendem a amamentar por mais tempo seus filhos (Tewabe et al., 2017).

A prática da amamentação pode sofrer interferência com o trabalho materno fora do lar ao passo que a presença materna no lar pode ser um fator de proteção (Alves et al.,2018) e no presente estudo prevaleceram genitoras donas de casa (76\%). A prática do AM tem sofrido variações ao longo dos anos, sobretudo, pelo fato de as mulheres terem conquistado seu espaço no mercado de trabalho, mas não deixaram de lado seu papel de mãe, entretanto, a amamentação ficou em segunda instância. A inserção da mulher no mercado de trabalho está intimamente atrelada à queda histórica do AM (Fernandes et al., 2018, Moimaz et al., 2020).

Os resultados da presente pesquisa mostraram que 76,7\% já tinham tido filhos anteriormente. Mulheres que já tiveram filhos anteriormente podem ter maior experiência e maturidade para amamentar os lactentes, considerando que essas mulheres possuem um maior conhecimento e consciência da importância do aleitamento materno, em comparação as mulheres que possuem somente um filho (Sousa et al., 2017). A multiparidade pode ser considerada fator de proteção para a amamentação, bem como a realização do pré-natal (Vieira et al., 2019).

O Ministério da Saúde por meio da Portaria SES-DF N³42 de 28.06.2017 recomenda pelo menos seis consultas durante o período gravídico intercaladas entre médico e enfermeiro (Governo Do Distrito Federal, 2017). Na presente pesquisa, $99,7 \%$ das participantes realizaram o pré-natal sendo que 65,4\% fizeram seis ou mais consultas. Mulheres que fazem o prénatal apresentam maior conhecimento sobre os benefícios da amamentação. A quantidade de consultas está ligada ao melhor resultado gestacional, prevenindo riscos (Alves et al., 2018).

O pré-natal é o momento mais oportuno para promover a prática da amamentação, desenvolvendo ações educativas direcionadas para a mulher, visando ao sucesso da amamentação. A equipe de saúde deve apoiar a mulher e serem facilitadores estando atentos às suas necessidades de orientações e cuidados (Santos et al., 2020).

Na presente casuística 76,7\% das mulheres já amamentaram na gravidez anterior e a prática do AME até seis meses esteve presente em 25\% dos casos, taxa considerada razoável segundo o Ministério da Saúde (12 a $49 \%$ razoável) (Brasil, 2015). Na região Nordeste a taxa de AME em 2020 era de 55,8\% (UFRJ, 2020). 
A Organização Mundial da Saúde preconiza que o AM exclusivo nos seis primeiros meses e após esse período, deve acontecer a complementação com outros alimentos e sendo mantido até os dois anos ou mais. Entretanto, a UNICEF aponta que apenas 44\% das crianças mundialmente recebem o AME (Who, 2017). Algumas dificuldades foram apontadas para o desmame precoce como fissura no mamilo, queixa de pouco leite, leite fraco e dor. Estudo realizado no interior de São Paulo também apontou que uma das principais queixas apresentadas pelas mães foi a quantidade insuficiente de leite, o que as levou a introduzir leite artificial como complemento da amamentação levando ao desmame precoce (Freitas et al., 2018).

Orientações sobre o manejo das dificuldades advindas da amamentação são de responsabilidade da equipe de saúde (Vilar et al., 2020), considerando que os mesmos tem papel preponderante no apoio ao aleitamento materno e na prevenção de dificuldades e no manejo dessa prática, promovendo uma amamentação efetiva (Santos et al., 2020).

Os problemas nos mamilos também foram apontados por 15,4\% das mulheres que citaram dificuldades para amamentar. Estudo realizado no interior do estado de São Paulo também encontrou que 13,73\% das mulheres citaram o ferimento mamilar como um dos problemas enfrentados bem como o leite insuficiente entre 33,33\% das pesquisadas (Freitas et al., 2018).

As lesões nos mamilos podem ser resultado de uma pega inadequada do bebê no momento de mamar, o que quase sempre está associado ao ingurgitamento mamário, que leva ao edema, fissuras mamárias e mastite, que causam sintomas dolorosos, o que pode ocasionar a interrupção da amamentação (Ministério da Saúde, 2015).

Sobre a queixa de leite fraco ou insuficiente, estudo realizado no interior do Amazonas apontou que 8,7\% das pesquisadas citaram dificuldades em amamentar como a baixa produção de leite $(8,7 \%)$ e rejeição do lactente ao leite materno $(13,0 \%)$. Estudos indicam que a queixa de leite fraco ou insuficiente são as mais frequentes o que leva ao desmame precoce (Alves et al., 2018; Freitas et al., 2018; Santos et al.,2020).

Fatores culturais podem levar a mulher a acreditar que seu leite é fraco ou insuficiente. Este equívoco pode estar vinculado à falta de conhecimento materno quanto aos valores do leite materno e ao fato de relacionarem o choro do bebê a falta de alimento, o que nem sempre é verídico (Araújo et al., 2020).

Fica cada vez mais evidente a importância do suporte dado pelos profissionais de saúde para o apoio e promoção do AM e o prolongamento do tempo da amamentação. Dessa forma há necessidade da implementação de ações educativas voltadas para a mulher, reforçando as orientações sobre essa temática bem como no manejo de dificuldades que possam acontecer (Souza et al, 2020).

Considerando as consequências indesejáveis tanto para a mãe como para o bebê, com o desmame precoce, é imperativo que medidas efetivas sejam adotadas pela equipe de saúde visando o prolongamento da amamentação, acolhendo as mulheres nessa etapa da vida (Almeida et al., 2019).

Como limitação encontrada pelos pesquisadores para a realização desse estudo pode-se apontar o fato de que as entrevistas foram realizadas nas enfermarias durante o puerpério imediato o que demandou um tempo maior para a coleta dos dados e pode ter causado algum constrangimento nas participantes em responder à pesquisa. Vale ressaltar ainda que a pesquisa aponta para uma realidade local e talvez esse cenário não pode ser estendido para outras realidades.

\section{Conclusão}

Os resultados encontrados mostraram que a maioria das participantes da pesquisa tinha idade entre 18 e 30 anos, eram casadas ou viviam em união estável, cursaram o ensino médio, eram donas de casa, tinham renda familiar entre um e dois salários mínimos, tinham dois filhos, fizeram seis ou mais consultas de pré-natal; no entanto, nem todas receberam orientações sobre aleitamento materno durante o pré-natal, a maioria já amamentou em gestação anterior e apenas uma pequena parcela 
amamentou exclusivamente até os seis meses de vida do bebê; ainda foi possível identificar que as principais dificuldades para amamentar citadas pelas mulheres foram queixa de pouco leite, fissura no mamilo, ter leite fraco e dor ao amamentar.

Evidencia-se então a importância do uso de atividades educativas voltadas para a amamentação pelos profissionais de saúde que prestam assistência ao pré-natal, parto e puerpério, visando contribuir para que as mães tenham condições de amamentar, reduzindo assim as taxas de desmame precoce.

\section{Agradecimentos}

Este estudo foi financiado pela Coordenação de Aperfeiçoamento de Pessoal de Nível Superior - Brasil (CAPES) - Finance Code 001.

\section{Referências}

Almeida, L. M., Costa, A. P., Santos, F. E. $\quad$ G., $\quad$ Medeiros, P. $\quad$ K., Oliveira, $\quad$ S. X., \& Nóbrega, M. M. (2019). Desmame precoce: principais causas e consequências para o bebê e para a mãe, uma revisão literária. Temas em saúde, 19(3), 214-228. https://temasemsaude.com/wp-content/uploads/2019/09/19313.pdf

Alves, T. V. \& Bezerra, M. M. M. (2020). Principais alterações fisiológicas e psicológicas durante o Período Gestacional. Id on Line Rev. Mult. Psic, 14(49), 114-126. DOI: $10.14295 /$ idonline.v14i49.2324

Alves, L. L., Cirino, I. P., Santos, M. S., Sousa, A. F.,\& Lima, L. H. (2018). O. Prevalência do aleitamento materno exclusivo e seus fatores de risco. Saúde e Pesquisa, 11(3), 527-534. DOI: https://doi.org/10.17765/1983-1870.2018v11n3p527-534

Alves, V. G. S., Mota, M. C., \& Pagliari, C. (2021). Características sociodemográficas relacionadas ao conhecimento dos benefícios do aleitamento materno. Revista Paulista de Pediatria, 39:e2020101, 1-9. http://dx.doi.org/10.1590/1984-0462/2021/39/2020101

Alves, V. G. S, Mota, M. C., \& Pagliari, C. (2021) Sociodemographic characteristics related to knowing the benefits of breastfeeding. Rev Paul Pediatr. 2;39:e2020101. doi: 10.1590/1984-0462/2021/39/2020101. eCollection 2021

Alves, J.S., Oliveira, M. I. C., \& Rito, R. V. F. (2018) Orientações sobre amamentação na atenção básica de saúde e associação com o aleitamento materno exclusivo. Ciência \& Saúde Coletiva, 23(4), 1077-1088. https://doi.org/10.1590/1413-81232018234.10752016

Amorim, T. V., Souza, Í. E. O., Moura, M. A. V, Queiroz, A. B. A., Salimena, A. M. O. (2017) Perspectivas de los cuidados de enfermería en el embarazo de alto riesgo: revisión integradora. Enfermería Global, 46: 515-529. http://dx.doi.org/10.6018/eglobal.16.2.238861.

Araújo, G. B., Fernandes, A. B., Oliveira, A. C. A., Gomes, E. G. R., Pereira, T. L., Leiliane, O. S., Silva, F. I., \& Abed, R. A. (2020). Contribuições do enfermeiro para a promoção do aleitamento materno. Brazilian Journal of health review, 3(3), 4841-4863. DOI: https://doi.org/10.34119/bjhrv3n3-070

Barbosa, E. O., Gomes, T. M. C., Mariano, A. F., Gonzaga, M. F. N., Tavares, S. S., Contini, I. C. P., \& Almeida, C. G. (2020). Assistência de enfermagem no pré-natal: um estudo de caso. Revista Saúde em Foco, 12, 349-356. https://portal.unisepe.com.br/unifia/wpcontent/uploads/sites/10001/2020/12/ASSIST\%C3\%8ANCIA-DE-ENFERMAGEM-NO-PR\%C3\%89-NATAL-UM-ESTUDO-DE-CASO-349-\%C3\%A0356.pdf

Brasil. Ministério da Saúde. Orientações direcionadas ao Centro de Operações de Emergências para o Corona-vírus (COE COVID-19), a serem adotadas pelo Sistema Único de Saúde (SUS) para a amamentação em eventuais contextos de transmissão de síndromes gripais. 2020. Nota Técnica N9/2020 DAPES/SAPS/MS. http://189.28.128.100/dab/docs/portaldab/documentos/nota tecnicaamamentacao92020DAPE SAPSMS03abr2020COVID-19.pdf

Brasil. Ministério da Saúde. Secretaria de Atenção à Saúde. Departamento de Ações Programáticas Estratégicas. Bases para discussão da Política Nacional de Promoção, Proteção e Apoio ao Aleitamento Materno. Brasília: Ministério da Saúde, 2017.

Brasil, Ministério da Saúde. Resolução No 466, DE 12 de dezembro de 2012. https://bvsms.saude.gov.br/bvs/saudelegis/cns/2013/res0466_12_12_2012.html

Fernandes, V. M. B., Santos, E. K. A., Zampieri, M. F. M., Gregório, V. R. P., Hernandes, M. J., \& Ribeiro, L. C. (2018). Condutas de gestores relacionadas ao apoio ao aleitamento materno nos locais de trabalho. Texto \& Contexto-Enfermagem, 27(3) e2560016.. http://dx.doi.org/10.1590/0104070720180002560016

Freitas, M. G., Werneck, A. L., \& Borim, B. C. (2018). Aleitamento materno exclusivo: adesão e dificuldades. Rev. Enferm. UFPE on line, 12(9), $2301-2307$. https://doi.org/10.5205/1981-8963-v12i9a234910p2301-2307-2018.

Harari, N., Rosenthal, M., Bozzi, V., Goeschel, L., Jayewickreme, T., Onyebeke, C., Griswold, M., \& Perez-Escamilla, R. (2018) Feasibility and acceptability of a text message intervention used as an adjunct tool by WIC breastfeeding peer counsellors: The LATCH pilot. Matern. Child Nutr., 14(1), e12488, 2018. doi: 10.1111/mcn.12488. Epub 2017 Aug 1

Lima, J. P., Cazola, L. H. O., \& Pícoli, R. P. (2017). A participação do pai no processo de amamentação. Cogitare Enfermagem, 22(1), e47846. DOI: https://doi.org/10.5380/ce.v22i1.47846

Margotti, E., \& Margotti, W. (2017). Fatores relacionados ao Aleitamento Materno Exclusivo em bebês nascidos em hospital amigo da criança em uma capital do Norte brasileiro. Saúde em Debate, 41(114), 860-871. DOI: 10.1590/0103-1104201711415 
Melo, D. S., Oliveira, M. H., \& Pereira, D. S. (2021). Progressos do Brasil na proteção, promoção e apoio do aleitamento materno sob a perspectiva do global breastfeeding collective. Revista Paulista de Pediatria, 39, 1-7. https://doi.org/10.1590/1984-0462/2021/39/2019296

Ministério da Saúde. (2015). Saúde da criança: Aleitamento materno e alimentação complementar (2 ${ }^{\mathrm{a}}$ ed.). Brasília, Brasil: https://bvsms.saude.gov.br/bvs/publicacoes/saude_crianca_aleitamento_materno_cab23.pdf.

Moimaz, S. A. S., Rós, D. T., Saliba, T. A., \& Saliba, N. A. (2020) Estudo quanti-qualitativo sobre amamentação exclusiva por gestantes de alto risco. Ciênc. Saúde Coletiva, 25(9), 2020. https://doi.org/10.1590/1413-81232020259.30002018

Moraes, I. C., Sena, N. L., Oliveira, H. K. F., Albuquerque, F. H. S., Rolim, K. M. C., Fernandes, H. I. V. M., \& Silva, C. N. (2020). Percepção sobre a importância do aleitamento materno pelas mães e dificuldades enfrentadas no processo de amamentação. Revista de Enfermagem Referência, 2, 1-7. https://pesquisa.bvsalud.org/portal/resource/pt/biblio-1125499

Organização Mundial De Saúde $\quad$ (OMS). $\quad$ Recomendações $\quad$ OMS em: $<$ https://www.paho.org/bireme/index.php?option=com_content\&view=article\&id= 213: opas-oms-preconiza-apoio-ao-aleitamento-materno-exclusivo-ateos-seis-meses \&Itemid=183\&lang=pt $>$.

Pereira, A. S., Shitsuka, D. M., Parreira, F. J., \& Shitsuka, R. [e-book]. Santa Maria. Ed. UAB/NTE/UFSM.: https://repositorio. ufsm.br/bitstream/handle/1/15824/Lic_Computacao_Metodologia-Pesquisa-Cientifica.pdf? sequence=1.

Reis, R. S., \& Rached, C. D. A. (2017). O papel do enfermeiro no acompanhamento de pré-natal de baixo risco utilizando a abordagem Centrada na pessoa gestante. International Journal of Health Management Review, 3(2), 1-32. file:///C:/Users/Flor/AppData/Local/Temp/125-68-1-SM-1.pdf DOI: $10.21902 /$ jhmreview.v3i2.125

Rocha, N. B., Garbin, A. J. I., Garbin, A. S., Saliba, O., \& Moimaz, S. A. S. (2013). Estudo longitudinal sobre a prática de aleitamento materno e fatores associados ao desmame precoce. Pesquisa Brasileira em Odontopediatria e Clínica Integrada, 13(4), 337-342. http://dx.doi.org/10.4034/pboci.2013.134.06

Rodrigues, A. P., Padoin, S. M. M., Aldrighi, J. P., Paula, C. C., \& Ximenes, L. B. (2016).Catacterização sociodemográfica e obstétrica de puérperas internadas em alojamento conjunto no Brasil. Ciência y enfermeria, 22(1), 113-126. http://dx.doi.org/10.4067/S0717-95532016000100010.

Santos, F. S., Souza, R. C., Candido, P. G. G., Santos, L. H., Pascoal, L. M., \& Santos Neto, M. (2020). Autoeficácia do aleitamento materno em puérperas de uma maternidade pública do nordeste brasileiro. Revista de Enfermagem do Centro-Oeste Mineiro, 10(e3910)1-9. https://doi.org/10.19175/recom.v10i0.3910

Santos, F. S., Lima, A. S., Viana, A. I. S., Santos, L. H., Santos Neto, M., Costa, A. C. P. J., \& Araújo, M. F. M. (2020). Aleitamento materno de crianças com microcefalia por zika vírus. Research, Society and Development, 9(6), e150963692. DOI: http://dx.doi.org/10.33448/rsd-v9i6.3692

Sousa, E. D. M., Santos, M. P., \& Santos, T. M. A. (2017). Prevalência de Aleitamento Materno em Crianças de 0 a 12 meses e seus Fatores Condicionantes. In: Congresso Internacional de Enfermagem. file:///C:/Users/Flor/AppData/Local/Temp/6224-22238-1-PB-1.pdf

Souza, E. F. C., Pina-Oliveira, A. A., \& Shimo, A. K. K. (2020). Effect of a breastfeeding educational intervention: a randomized controlled trial. Revista Latino-Americana de Enfermagem, 30(28), e3335. doi: 10.1590/1518-8345.3081.3335. eCollection 2020.

Tewabe, T., Mandesh, A., Gualu, T., Alem, G., Mekuria, G., \& Zeleke, H. (2015). Exclusive breastfeeding practice and associated factors among mothers in Motta town, East Gojjam zone, Amhara Regional State, Ethiopia, 2015: a cross-sectional study. International Breastfeeding Journal, 12(12), 1-7. DOI $10.1186 / \mathrm{s} 13006-017-0103-3$

Universidade Federal Do Rio De Janeiro (UFRJ). Estudo Nacional de Alimentação e Nutrição Infantil - ENANI-2019: Resultados preliminares - Indicadores de aleitamento materno no Brasil. UFRJ: Rio de Janeiro, 2020. 9 p.

Vieira, F. S., Costa, E. S., Sousa, G. C., Oliveira, T. M. P., \& Neiva, M. J. L. M. (2019). Childbirth Influence Towards the Weaning During Puerperium Period. Revista de Pesquisa: Cuidado é Fundamental, 11(2), 425-431. DOI: https://doi.org/10.9789/2175-5361.2019.v11i2.425-431

Vilar, T. M., Oliveira, I. K. F., Monteiro, N. V. N., Araújo, F. Y. G., \& Carvalho, C. M. R. G. (2020). Educação em saúde e direito: em busca da proteção do aleitamento materno e dos direitos das gestantes em uma maternidade pública. Research, Society and Development, 9(1), e22911552. doi.org/10.33448/rsdv9i1.1552

Vila-Candel, R., Soriano-Vidal, F. J., Murillo-Llorente M., Pérez-Bermejo, M., \& Castro-Sánchez, E.. (2019) Maintenance of exclusive breastfeeding after three months postpartum: An experience in a health department of a Valencian Community. Aten Primaria, 51(2), 91-8. doi: 10.1016/j.aprim.2017.09.002

World Health Organization. (2017). Guideline: protecting, promoting and supporting breastfeeding in facilities providing maternity and newborn services. World Health Organization, https://apps.who.int/iris/handle/10665/259386 